\title{
Ion temperature fluctuations in the ASDEX Upgrade scrape-off layer
}

\author{
M. Kočan ${ }^{1}$, F. P. Gennrich ${ }^{2}$, A. Kendl ${ }^{2}$, H. W. Müller ${ }^{1}$ and the ASDEX Upgrade Team \\ ${ }^{1}$ Max-Planck-Institut für Plasmaphysik, EURATOM Association, Boltzmannstr. 2, D-85748 Garching, Germany \\ ${ }^{2}$ Institute for Ion Physics and Applied Physics, Association Euratom-ÖAW, University of Innsbruck, \\ Technikerstraße 25, A-6020 Innsbruck, Austria \\ E-mail: martin.kocan@ipp.mpg.de
}

\begin{abstract}
The ion temperature in turbulent plasma filaments $T_{i f i l}$ is measured by a retarding field analyzer during L-mode discharges in the ASDEX Upgrade tokamak. At $2 \mathrm{~cm}$ outside the separatrix $T_{i \text { fil }} \approx 80-110 \mathrm{eV}$, which is 3-4 times that of the background ions and $50-70 \%$ of the ion temperature at the separatrix. $T_{i f i l}$ is reproduced by a fluid model of the parallel filament transport assuming the radial filament propagation speed of $400-1000 \mathrm{~m} \mathrm{~s}^{-1}$ in the near scrape-off layer, consistent with earlier experimental estimates. The conditional sampling used in experiment to measure $T_{i \text { fil }}$ is tested on artificial time series obtained from a gyrofluid turbulence simulation.
\end{abstract}

PACS: 52.25.Xz, 52.35.Ra, 52.55.Fa, 52.65.-y, 52.65.Tt, 52.70.Ds

\section{Introduction}

Turbulence is responsible for a large fraction of the convective transport across the tokamak Scrape-Off Layer (SOL) [1,2]. Turbulent transport is ascribed to radial convection of the field-aligned plasma filaments (or blobs, as seen in the plane perpendicular to the magnetic field B) ejected near the separatrix on the plasma outboard side [3-6]. Propelled by interchange motions [7], the filaments convect plasma to the wall with much higher density than the background plasma density [8]. In ITER, the filament-wall interaction can cause unwanted erosion and re-deposition of the beryllium plasma facing components (PFCs). 
These processes can affect the lifetime of the first wall PFCs and the in-vessel tritium inventory $[9,10]$. It has been shown in [8] that filament electrons can thermalize with the background plasma within a short radial distance from the separatrix. However, less mobile ions in the filaments could carry a considerable fraction of their initial energy to the first wall and increase the sputtering from the main chamber PFCs. The measurements of the ion temperature in the filaments, $T_{i f i l}$ would also provide useful background information for turbulence modelling. Unfortunately, with the exception of some preliminary measurements on CASTOR [11] and ISTTOK [12], $T_{i f i l}$ is practically unknown.

This article reports on measurements of $T_{i f i l}$ in the L-mode plasma in the ASDEX Upgrade (AUG) tokamak [13] using a retarding field analyzer (RFA). The measured $T_{i \text { fil }}$ is compared with the background $\left(T_{i}\right)$ and the separatrix $\left(T_{i s e p}\right)$ ion temperatures. A simple fluid model of the filament parallel transport in the SOL [14] is employed to interpret RFA measurements and estimate the characteristic range of the filament radial propagation speeds, $v_{r}$.

Section 2 outlines the experimental setup and the RFA technique. The RFA data from which $T_{i}$ and $T_{i f i l}$ are obtained, are discussed in Section 3. In the same section, $v_{r}$ is estimated by combining the RFA data with the parallel loss model. In Section 4 we examine the reliability of the conditional averaging used to obtain $T_{i f i l}$ on artificial RFA measurements generated by the gyrofluid turbulence code GEMR. A summary is given in Section 5.

\section{Experimental set-up}

ASDEX Upgrade is a divertor tokamak with a tungsten-coated main chamber wall and divertor [13]. RFA measurements of $T_{i f i l}$ were obtained in the lower single null L-mode discharge (\#26190) with the magnetic equilibrium shown in figure 1. The plasma current $I_{p}=$ 0.8 MA, toroidal magnetic field $B_{t}=2.5 \mathrm{~T}$ (with the ion $\mathbf{B} \times \nabla B$ drift direction towards the lower X point), safety factor of $95 \%$ flux surface $q_{95}=5.6$, major radius $R=1.68 \mathrm{~m}$, ohmic

power $P_{o h m}=0.4 \mathrm{MW}$ and the electron cyclotron resonance heating of $P_{E C R H}=0.7 \mathrm{MW}$. Line-integrated core plasma density $\bar{n}_{e}=4 \cdot 1 \cdot 10^{19} \mathrm{~m}^{-2}$ (44\% of the Greenwald density). A plasma density at the separatrix, $n_{\text {esep }} \cong(0.5-1) \cdot 10^{19} \mathrm{~m}^{-3}$ was measured with a lithium beam 
diagnostic and $T_{i s e p}=150 \mathrm{eV}$ from charge exchange recombination spectroscopy. Because of the ambiguity in the electron temperature measurements at the separatrix by the electron cyclotron emission diagnostic in discharge \#26190 (most likely due to the low optical thickness of the edge plasma) we assume $T_{\text {esep }}=50 \mathrm{eV}$. This corresponds to $T_{\text {esep }}$ values measured previously in the AUG L-mode discharges by the Thomson scattering diagnostic [15] (in the present discharge, a large data scatter makes the Thomson scattering measurements at the separatrix effectively useless). The discharge is characterized by the parallel connection length $L_{/ /} \cong q_{95} \pi R \approx 30 \mathrm{~m}$ and the parallel separatrix collisionality $v_{e}^{*} \approx L_{/ /} / \lambda_{e e} \approx 6-12$, with the electron-electron collisional mean free path $\lambda_{e e} \approx 10^{16} T_{\text {esep }}^{2} / n_{\text {esep }}\left(\mathrm{m}, \mathrm{eV}, \mathrm{m}^{-3}\right)$. The radial distance between the separatrix and the outboard limiter, mapped to the outboard midplane, is $6.6 \mathrm{~cm}$.

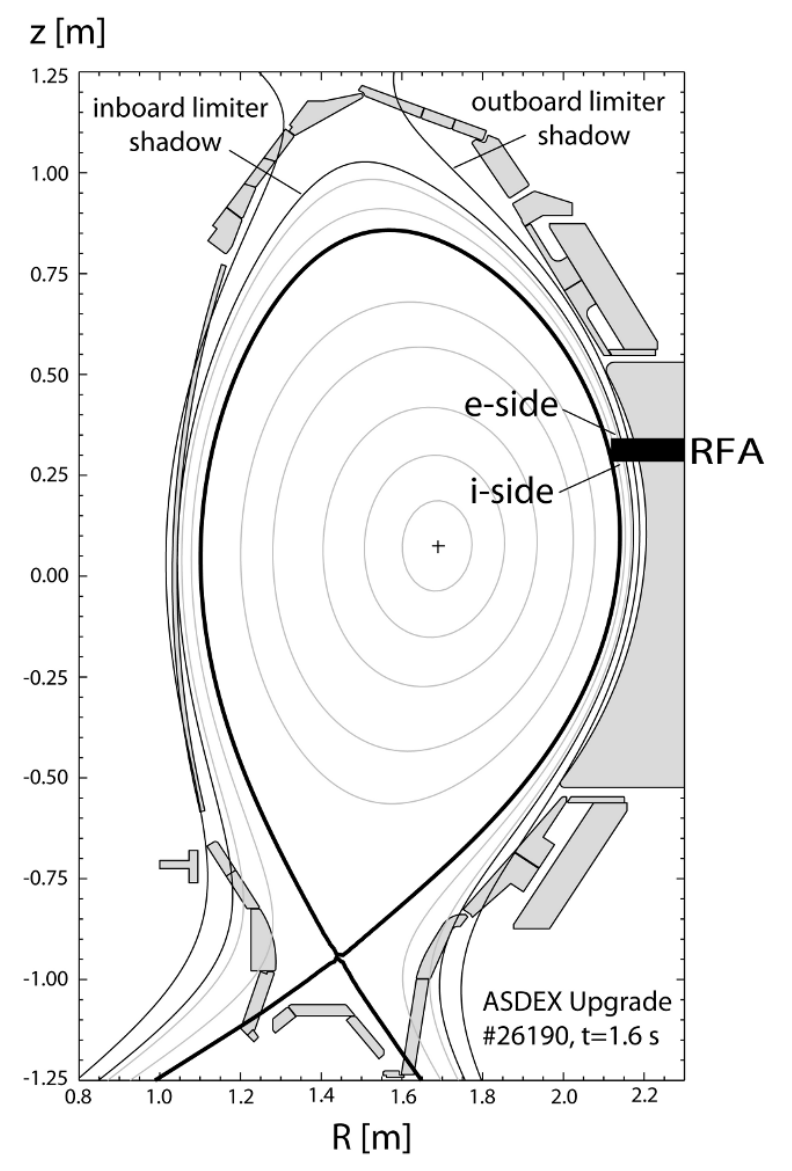

Figure 1. Poloidal cross-section of $A U G$ in discharge \#26190. i-side / e-side indicates the side of a bidirectional RFA. The RFA axis is aligned with the local magnetic field. The i-side (e-side) RFA faces the outer (inner) divertor along the magnetic field lines.

As shown in Fig. 1, the horizontally reciprocating RFA is installed $31 \mathrm{~cm}$ above the outer midplane. The probe head, described in more detail in $[16,17]$, has an outer diameter of 
$6.2 \mathrm{~cm}$. The RFA sensors are located $15 \mathrm{~mm}$ behind the probe tip. The probe is aligned with $\mathbf{B}$ so that the RFA is sensitive to the parallel-to-B velocity of incident ions. The RFA consists of two identical analyzers mounted in a Mach probe arrangement, monitoring the plasma from both directions along the magnetic field lines. As shown in Fig. 1, the "i-side" of the RFA faces in the direction towards the outer divertor and the "e-side" of the RFA is looking along field lines into the inner divertor. The i-side / e-side denote the side of the RFA intercepting the ion / electron drift directions. As depicted in Fig. 2, each analyzer consists of a set of semi-permeable grids and a collector separated from the plasma by a thin plate into which a narrow slit is cut. The slit plate is biased negatively $\left(V_{s p}=-100 \mathrm{~V}\right)$ to repel most of the incident electrons back into the plasma and to measure the ion saturation current density $j_{s a t}=I_{s p} / A_{s p} \quad\left(I_{s p}\right.$ and $A_{s p}=16 \mathrm{~mm}^{2}$ being respectively the slit plate current and the collecting area). A narrow slit, cut in the slit plate, admits enough ions to produce a measurable current $I_{c}$ on the collector. Collector currents down to $\sim 0.3 \mu \mathrm{A}$ can be measured without any significant noise problem. Ions transmitted through the slit encounter a grid, labelled as "grid 1", to which a positive swept voltage $V_{g 1}=0 \rightarrow 270 \mathrm{~V}$ is applied with the frequency $f_{g 1}=1.5 \mathrm{kHz}$. The ions that have enough kinetic energy to overcome $V_{g 1}$ proceed to a second grid, labelled as "grid 2", biased to $V_{g 2}=-180 \mathrm{~V}$, and placed between grid 1 and the collector. Grid 2 is used to repel any remaining incident electrons that are energetic enough to penetrate $V_{s p}$, as well as to suppress secondary electrons emitted from the collector or from the rear of the slit plate by ion impact. Note that a third grid is placed just behind the slit plate to render the electric field between the slit plate and grid 1 as planar as possible. The same voltages are applied to both analyzers. Signals are measured at $2 \mathrm{MHz}$ sampling rate. During the reciprocation in the discharge \#26190, the RFA was maintained for $10 \mathrm{~ms}$ at the midplane separatrix distance $\Delta r_{\text {sep }}=21 \mathrm{~mm}$, which is outside the shadow of both the inboard and the outboard limiters. 


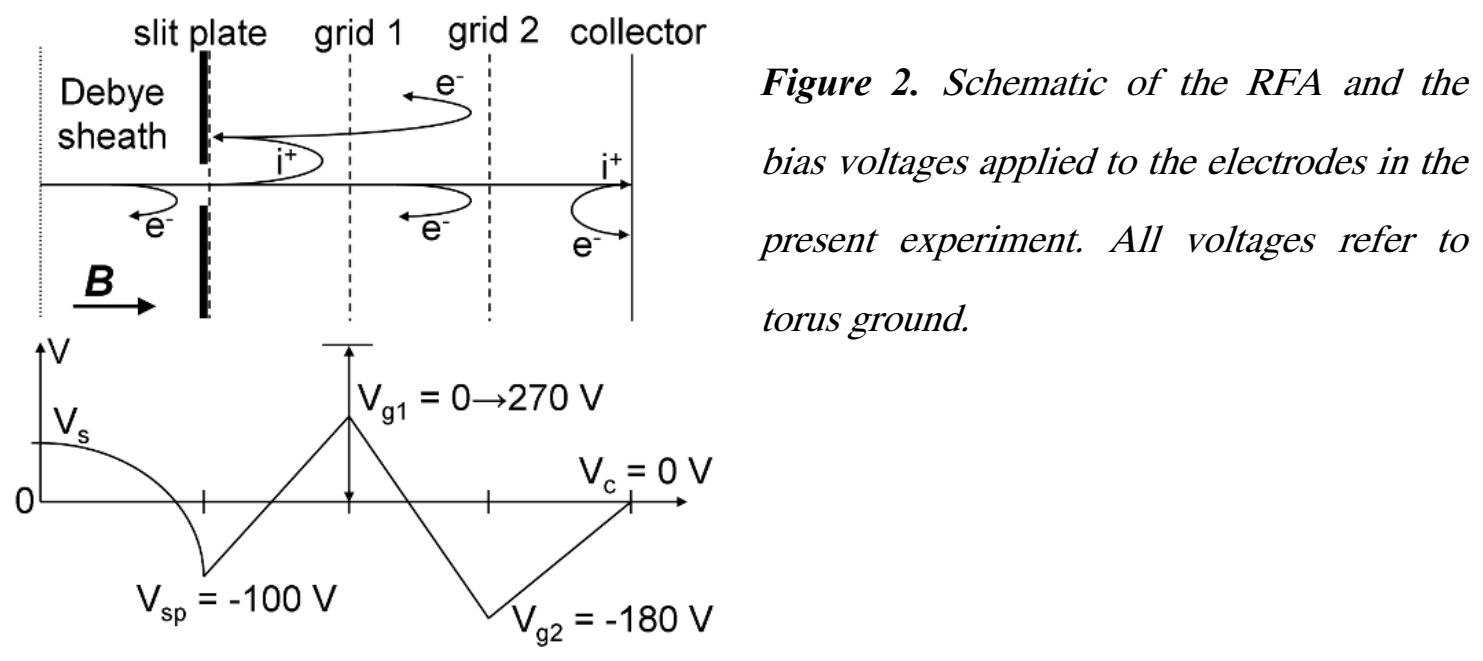

\section{Experimental results}

\section{1. RFA time traces in L-mode}

Figure 3 illustrates the RFA time traces recorded at $\Delta r_{\text {sep }}=21 \mathrm{~mm}$. On average, the currents measured by the i-side RFA are much larger, indicating that the unperturbed plasma flow at the probe location is directed towards the inner divertor, which will be discussed later. However, most striking is the difference in the dynamic behavior of the time traces sampled by each RFA. Intermittent positive bursts are observed on the slit plate as well as the collector of the i-side RFA, even during the periods of high $V_{g 1}$, represented by shaded areas in Fig. 3 . Fluctuation amplitudes of $j_{\text {sat }}^{i-s i d e}$ of more than 3 times the standard deviation $\sigma$ above the time-averaged mean are measured every $0.5 \mathrm{~ms}$ on average and, as shown in Fig. 4, they last about $15 \mu \mathrm{s}$. The conditionally averaged time evolution of these bursts is asymmetric around the maximum, with a steep front followed by a trailing wake. This is consistent with the predicted dynamical evolution of the filament due to interchange motions [7] as well as with earlier experimental observations (e.g. $[2,18-20]$ ). The currents measured by the e-side RFA are far less intermittent. The dynamical behavior of $j_{\text {sat }}$ measured by each RFA is reflected in the probability distribution functions plotted in Fig. 5 and the statistical moments of the same signals compiled in Table 1 . The fluctuations of $j_{\text {sat }}^{i-s i d e}$ are positively skewed and leptokurtic, whilst those of $j_{\text {sat }}^{e-s i d e}$ are close to a normal distribution. In Table 1 , the excess kurtosis $K$ is evaluated as $\mu^{4} / \sigma^{4}-3$ (where $\mu^{4}$ is the fourth central moment and $\sigma$ is the standard 
deviation) so that both $K=0$ and the skewness, $S=\mu^{3} / \sigma^{3}=0$, for the normal distribution. Statistical moments of $j_{\text {sat }}^{i-s i d e}$ are similar to those previously measured in AUG (and earlier e.g. in $[1,2,20])$ by a Langmuir probe pin inserted at similar $\Delta r_{\text {sep }}$ and at the same poloidal and toroidal position as the RFA [21]. This is easily understood since, according to the present results, the total current measured by the pin should be dominated by ions collected by the iside of the pin.

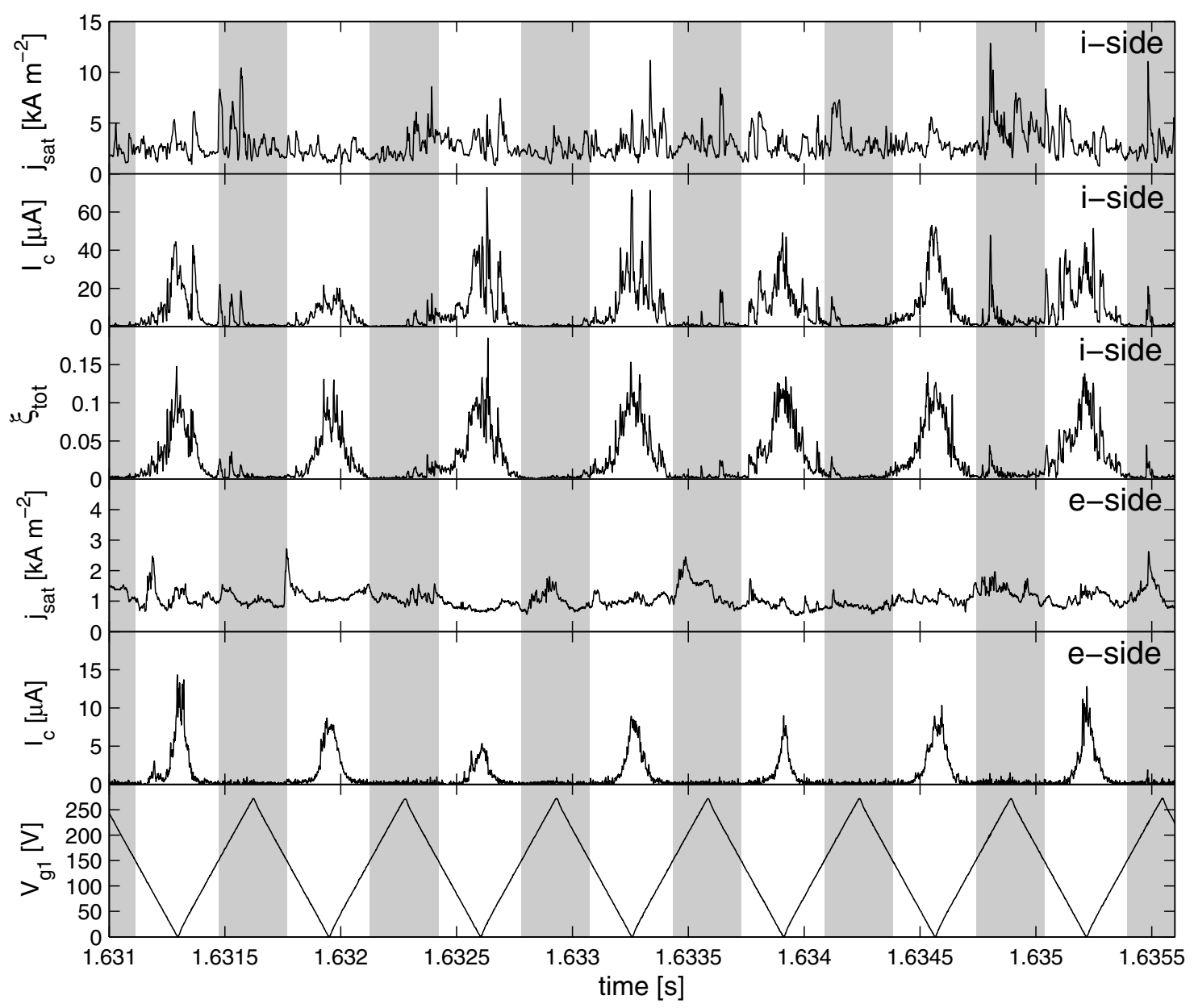

Figure 3. Time evolution of the ion current density and the collector current measured by $R F A$ at $\Delta r_{\text {sep }}=21 \mathrm{~mm}$. $i$-side / e-side denotes the side of the RFA indicated in Fig. 1. Also shown is the time evolution of the total ion transmission factor of the $i$-side $R F A$, $\xi_{\text {tot }}$ (see text) and the ion repelling voltage applied to both RFAs. Shaded areas correspond to periods of $V_{g 1}>150 \mathrm{~V}$. 


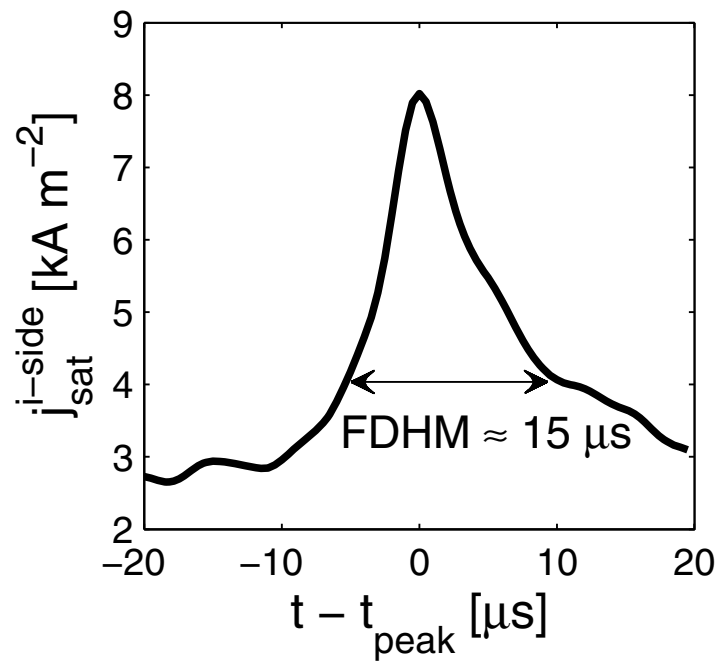

Figure 4. Time evolution of the largeamplitude bursts $(>3 \sigma)$ of $j_{\text {sat }}^{i-\text { side }}$ obtained by averaging 35 events measured at $\Delta r_{\text {sep }}=$ $21 \mathrm{~mm}$. Also indicated is the full duration at half maximum.

Strong poloidal asymmetry of the radial particle and energy transport was previously observed in the divertor as well as limiter tokamaks (e.g. [3, 4, 6, 22-24] and references therein) and stellarators [25]. These observations indicate that the filaments are expelled into the SOL in the vicinity of the outboard midplane by the ballooning-type instability. As the filaments propagate outwards in the SOL, they extend along the field lines, driving parallel flows $[3,5]$. Since the probe is located above the outboard midplane, it seems obvious that the intermittent current bursts are observed mainly by the i-side RFA which faces most filaments as they expand along the flux tubes. The same conclusion has been reached elsewhere [26, 27].

As can be seen from Fig. 3, the bursts of $I_{c}^{i-s i d e}$ observed at high $V_{g 1}$ when the collector current is normally absent, are, without exception, synchronized with the bursts of $j_{\text {sat }}^{i-s i d e}$. This indicates that the filament ions are more energetic than those of the surrounding background plasma, which will be addressed in Sec. 3.3. In order to demonstrate this more clearly, Fig. 3 shows the time evolution of the total ion transmission factor of the i-side RFA, evaluated as $\xi_{\text {tot }}=I_{c}^{i-s i d e} /\left(j_{\text {sat }}^{i-s i d e} A_{\text {slit }}\right)$, where $A_{\text {slit }}=0.1 \mathrm{~mm}^{2}$ is the slit area. If the bursts of $I_{c}^{i-s i d e}$ observed at high $V_{g 1}$ were only due to increase of the incoming ion flux in the filaments, $\xi_{\text {tot }}$ would not change when the filaments strike the probe. The fact that $\xi_{\text {tot }}$ increases as the filaments pass the RFA sensors indicates that ions in the filaments carry higher energies than the background plasma ions. 

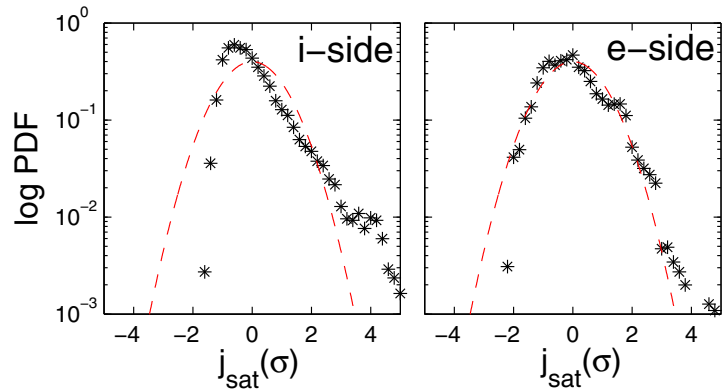

Figure 5. PDF of $j_{\text {sat }}$ measured at $\Delta r_{\text {sep }}=21 \mathrm{~mm}$, compared to normal distribution (dashed). $i$-side / e-side refers to the side of the RFA indicated in figure 1.

\begin{tabular}{lll}
\hline RFA & i-side & e-side \\
$\bar{j}_{\text {sat }}\left[\mathrm{kA} \mathrm{m}^{-2}\right]$ & 2.79 & 1.10 \\
$j_{\text {sat rms }} / \bar{j}_{\text {sat }}$ & 1.10 & 1.04 \\
skewness & 2.00 & 0.69 \\
excess kurtosis & 6.61 & 0.71 \\
\hline
\end{tabular}

Table 1. Mean value, relative fluctuation level, skewness and excess kurtosis of the ion current density measured by the $i$-side and the e-side $R F A$ at $\Delta r_{\text {sep }}=21 \mathrm{~mm}$.

\subsection{Background ion temperature}

When the parallel collisionality is high, it is a standard practice to assume that in the SOL the ions have a drifting Maxwellian distribution of parallel speeds $f\left(v_{/ /}\right)$and to equate the RFA e-folding voltage to the effective ion temperature $T_{i^{*}} \approx-\left(d \ln I_{c} / d V_{g 1}\right)^{-1}$ [28]. At $\Delta r_{\text {sep }}=21 \mathrm{~mm}$, the electron density measured by the lithium beam diagnostic $n_{e} \cong(0.7-2.5) \cdot 10^{18} \mathrm{~m}^{-3}$. We anticipate $T_{e} \approx 10 \mathrm{eV}$ obtained in this section, so that $v_{e}^{*} \approx L_{/ /} / \lambda_{e e} \approx 21-75$ at $\Delta r_{\text {sep }}=21 \mathrm{~mm}$. Under such conditions, it is reasonable to assume that ions are thermalized and use the standard RFA model (e.g. Ref. [28]) to obtain $T_{i^{*}}$. However, from the two RFA collector currents, $I_{c}^{i-s i d e}$ is contaminated by intermittent bursts associated to ions in the filaments, which, as will be shown later, have characteristic energies higher than those of the background ions. One could try to extract the inter-burst periods of $I_{c}^{i-s i d e}$ and estimate the effective temperature $T_{i^{*}}^{i-s i d e}$. However, we ignore the $\mathrm{i}$-side measurements and use $I_{c}^{e-s i d e}$ (which is almost unaffected by filament ions) to infer $T_{i^{*}}^{e-s i d e}$. 


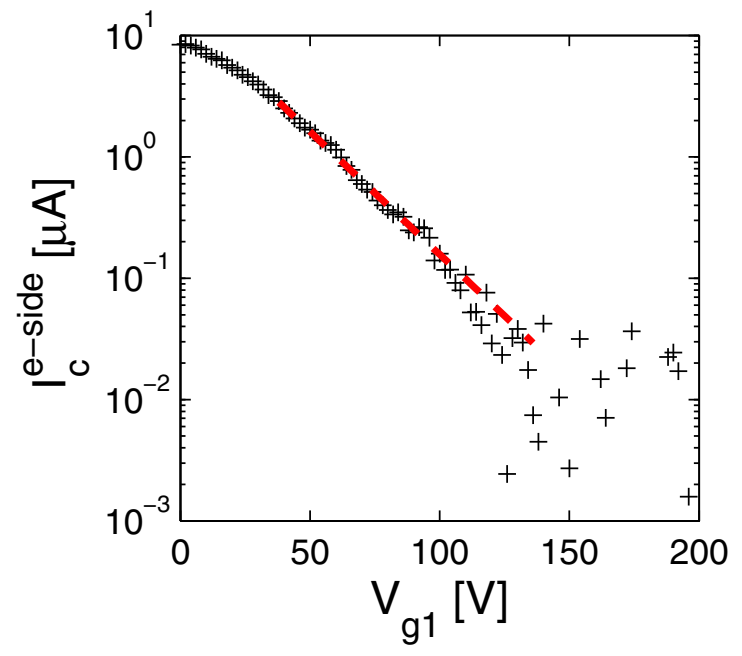

Figure 6. I-V characteristic measured by the e-side RFA at $\Delta r_{\text {sep }}=21 \mathrm{~mm}$. The slope of the exponential fit gives the effective ion temperature $T_{i^{*}}^{\text {down }}=23 \pm 4 \mathrm{eV}$.

Figure 6 shows the current-voltage $(I-V)$ characteristic $\left(I_{c}^{e-s i d e}\right.$ plotted against $\left.V_{g 1}\right)$ obtained by averaging the data sampled by the e-side RFA at $\Delta r_{\text {sep }}=21 \mathrm{~mm}$. Note the rounding of the $I-V$ characteristic at lowest $V_{g 1}$ which results from the distortion of the lowenergy part of the original drifting Maxwellian distribution in the quasi-neutral pre-sheath in front of the probe. This effect was addressed in detail in Refs. [29, 30]. Also plotted in Fig. 6 is the exponential fit to the high-energy part of the characteristic which yields $T_{i^{*}}^{e-s i d e}$. The same fitting procedure, applied to $I-V$ characteristics measured at different $\Delta r_{\text {sep }}$, yields the radial profile of the ion temperature shown in Fig. 7. Also plotted in Fig. 7 is the profile of $T_{e}$ measured in the similar L-mode discharge \#26148. In this discharge the slit plate was swept at $1 \mathrm{kHz}\left(V_{s p}=-140 \rightarrow 30 \mathrm{~V}\right)$ and $T_{e}$ was obtained using the standard magnetized Langmuir probe theory [31]. The values of $T_{e}$ measured by the RFA in the present experiment agree with the electron temperature measured earlier in the SOL of a similar L-mode discharge in AUG by the Thomson scattering diagnostic [15]. 


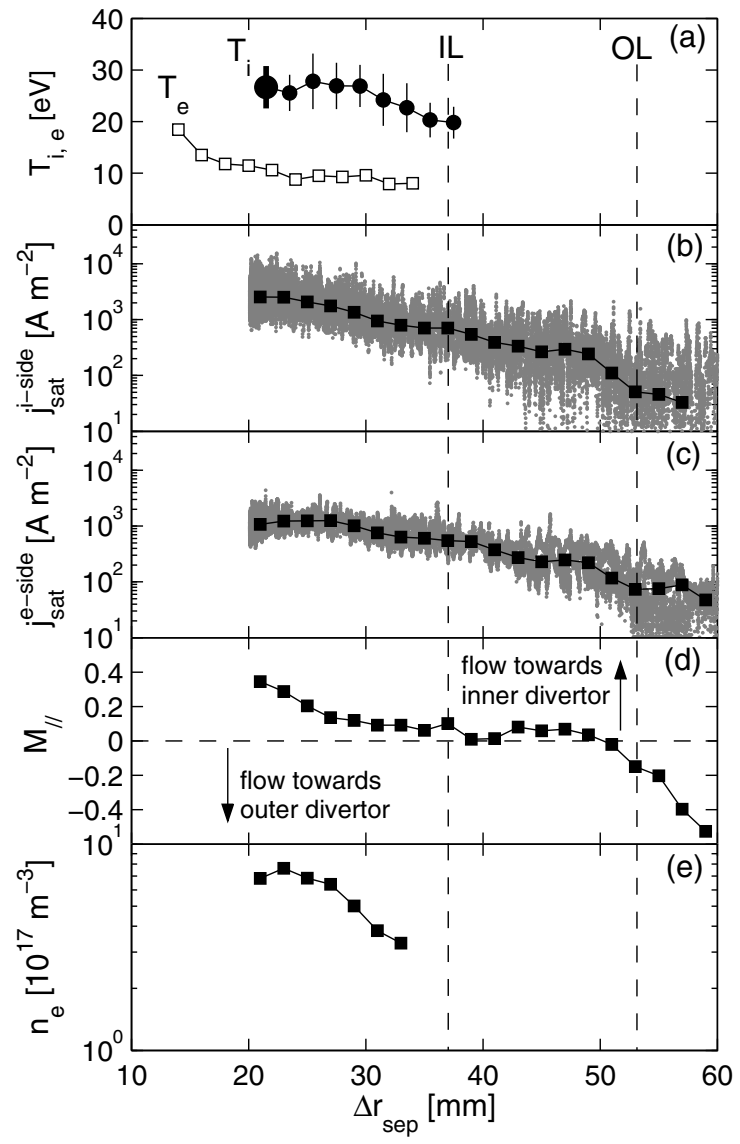

Figure 7. Radial profiles of selected background plasma parameters measured in the SOL by the RFA. (a) Ion and electron temperatures ( $T_{i}$ obtained from the exponential fit to the I-V characteristics plotted in figure 6 is highlighted), ion current density measured by (b) i-side and (c) e-side RFA slit plates (full symbols: binned median values), (d) parallel Mach number estimated from the binned median values of $j_{\text {sat }}$ and (e) electron density. IL/OL: inboard/outboard limiter shadow.

A background plasma flow introduces an asymmetry in $T_{i^{*}}$ measured by each side of the probe so that the unperturbed background ion temperature $T_{i} \neq T_{i^{*}}$ [29]. The ion temperature asymmetry has the same qualitative dependence on flow as the asymmetry of $j_{s a t}$ i.e. $T_{i^{*}}$ and $j_{\text {sat }}$ are both higher on the side of the probe facing the plasma flow [29-33]. With reasonable accuracy, $T_{i}$ can be approximated by $\left(T_{i^{*}}^{i-s i d e}+T_{i^{*}}^{e-s i d e}\right) / 2$ [29]. Since $T_{i^{*}}^{i-s i d e}$ cannot be measured in the case here, in order to estimate $T_{i}$ we use Fig. 7 from Ref. [29] which predicts $T_{i^{*}}^{i-s i d e} / T_{i^{*}}^{e-s i d e}$ as a function of the plasma flow velocity normalized to the cold ion sound speed $U_{d}=V_{/ /} / c_{e}$ where $c_{e}=\sqrt{e T_{e} / m_{i}}$. The parallel background plasma flow velocity normalized to ion sound speed $M_{/ /}=V_{/ /} / c_{s} \cong 0.4 \ln \left(j_{\text {sat }}^{i-\text { side }} / j_{\text {sat }}^{e-\text { side }}\right)$ [34], with $c_{s}=\sqrt{e\left(T_{i}+T_{e}\right) / m_{i}}$, estimated from the binned median values of $j_{\text {sat }}^{i-s i d e}$ and $j_{\text {sat }}^{e-s i d e}$ plotted in Fig. 7 (median filtering is used in order to reject intermittent bursts of $j_{\text {sat }}^{i-s i d e}$ ). As can be seen in Fig. 7, the parallel background plasma flow is directed towards the inner divertor for $\Delta r_{\text {sep }}<38 \mathrm{~mm}$, roughly stagnates for $38<\Delta r_{\text {sep }}<50 \mathrm{~mm}$, and is directed towards the outer divertor for $\Delta r_{s e p}>50 \mathrm{~mm}$ (in this region, however, the measurement of $M_{/ /}$become less 
reliable due to low-level slit plate currents). Also plotted in Fig. 7 is the background plasma electron density $n_{e}=\sqrt{j_{\text {sat }}^{i-\text { side }} j_{\text {sat }}^{e-\text { side }}} /\left(0.35 e c_{s}\right)[35,36]$ obtained from the binned median values of $j_{\text {sat }}^{i-\text { side }}$ and $j_{\text {sat }}^{e-s i d e}$. Estimated $M_{/ /} \approx 0.3$ at $\Delta r_{\text {sep }}=21 \mathrm{~mm}$ corresponds to $U_{d}=M_{/ /} \sqrt{1+T_{i} / T_{e}} \approx 0.5-0.6 \quad$ (with $T_{i} / T_{e}=2.5$ ) for which Ref. [29] predicts $T_{i^{*}}^{i-s i d e} / T_{i^{*}}^{e-s i d e} \approx 1.3$. Therefore, the unperturbed background ion temperature in Fig. 7 is obtained as $1.15 \cdot T_{i^{*}}^{e-s i d e}$, which is likely closer to $T_{i}$ than the measurements of $T_{i^{*}}^{e-s i d e}$ alone.

We note that $T_{i}>T_{e}$ seen in Fig. 7 was observed in L-mode plasmas in a number of limiter and divertor tokamaks ([37] and references therein). In the divertor configuration, $T_{i}>T_{e}$ in the SOL can be explained by higher parallel conductivity of electrons compared to that of less mobile ions, so that electron fluid experiences stronger cooling by parallel losses to a divertor [38].

\section{3. Filament ion temperature}

At least in theory, the most straightforward way to measure the ion temperature on the filament time scale by a RFA is to sweep $V_{g 1}$ with a frequency $f_{g 1}$ higher than the rate of change of the filament ion current, which is several $100 \mathrm{kHz}$. This is, however, beyond the capability of the RFA diagnostic set up in AUG, featuring $f_{g 1}$ up to several tens of $\mathrm{kHz}$.

In the present experiment, $T_{i \text { fil }}$ is obtained using the conditional averaging technique similar to that described in [39]. The peaks of the ion current density $j_{\text {sat fil }}$ and the corresponding collector current $I_{c \text { fil }}$ are selected from the time traces measured by the i-side RFA at $\Delta r_{\text {sep }}=21 \mathrm{~mm}$ (within the time resolution of the current measurements, the peaks of $j_{\text {sat }}^{i-s i d e}$ and $I_{c}^{i-s i d e}$ are well aligned). Excluded from the analysis are the peaks in close proximity to each other or those below the detection threshold which is set to $1 \sigma$ above the time-averaged mean, $\bar{j}_{\text {sat }}+\sigma\left(j_{\text {sat }}\right)=4.3 \mathrm{kA} \mathrm{m}^{-2}$. Filaments are sorted into groups characterized by similar $j_{\text {sat fil }} \pm 0.5 \mathrm{kA} \mathrm{m}^{-2}$. For each group, the filament $I$ - $V$ characteristic is obtained by plotting $I_{c f i l}$ against the corresponding $V_{g 1}$ as illustrated in Fig. 8 . Approximately exponential decay of $I_{c f i l}$ with increasing $V_{g 1}$, plus the relatively high value of $v_{e} *$ (Sec. 2), indicate that the parallel velocities of filament ions may be reasonably well described by a Maxwellian distribution with the characteristic temperature $T_{i \text { fil }}$. As illustrated 
in Fig. 8, $T_{i f i l}$ is obtained from the exponential fit to the $I-V$ characteristic. Included in the fit are only the filaments measured for $V_{g 1}>V_{g 1 \text { min }}$ with arbitrary $V_{g 1 \text { min }}=70 \mathrm{~V}$ - a range of voltages in which the measured $I_{c \text { fil }}$ apparently decreases with increasing $V_{g 1}$. The value of $V_{g 1 \text { min }}$ involves a trade-off: one wishes to include a sufficient number of data points for the fit, but to discard from the fit a non-decaying part of the $I-V$ characteristic due to ion acceleration in the sheath potential, as will be addressed in more details in the following section. Test trials with $V_{g 1 \min }=50-90 \mathrm{~V}$ yield the same $T_{i f i l}$ within about $30 \%$. The variation seems to be mainly due to a relatively low number of filaments in each $I-V$ characteristic.

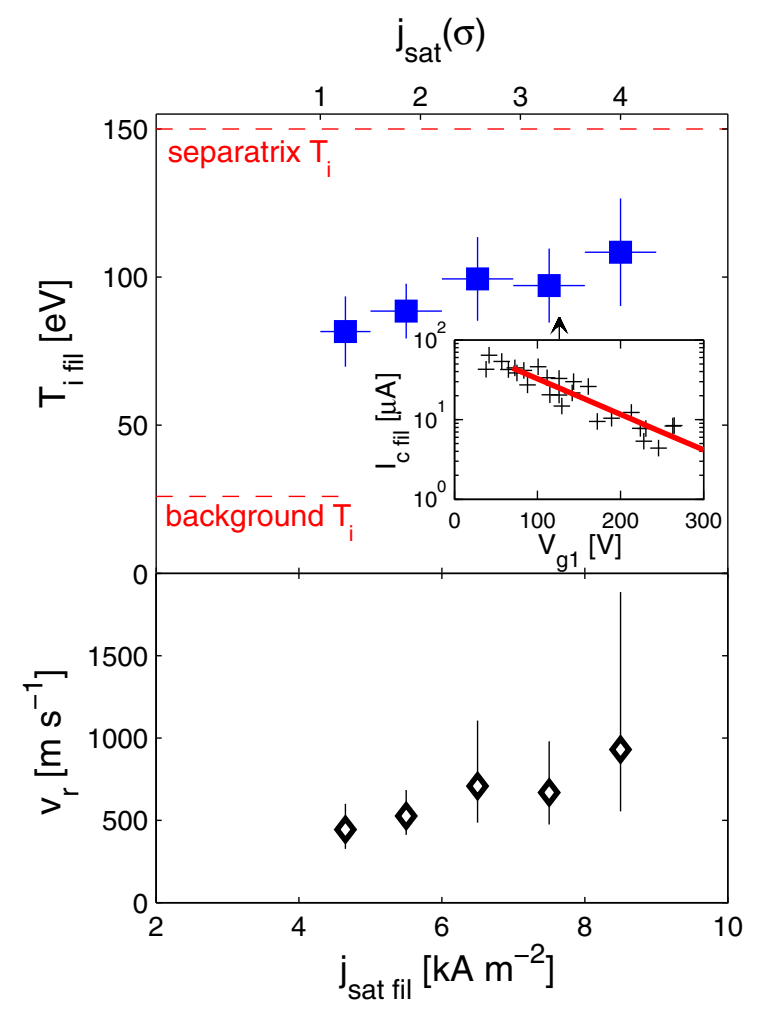

Figure 8. Top: Ion temperature in turbulent filaments measured by the RFA and plotted against the peak filament ion current density. $j_{\text {sat }}(\sigma)$ is the level of $j_{\text {sat }}$ above the timeaveraged mean, normalized to standard deviation. Dashed: $T_{i \text { sep }}$ and the ion temperature of the background plasma measured at the same $\Delta r_{\text {sep }}$ as $T_{i \text { fil }}$ Inset panel: the filament ion I-V characteristic for $j_{\text {sat fil }}=7-8 \mathrm{kA} \mathrm{m}^{-2}$ with the exponential fit. Below: $v_{r}$ required in the fluid simulations of the parallel filament transport to match $T_{i f i}$ measured by the RFA. 
Shown in Fig. 8 is $T_{i f i l}$ as a function of $j_{\text {sat fil }}$. The error bar of $T_{i f i l}$ equals to the confidence interval of the fit to the filament $I-V$ characteristic. The horizontal bars indicate the range of $j_{\text {sat fil }}$ of the filaments included in the $I-V$ characteristic. Horizontal dashed lines indicate the background ion temperature measured at $\Delta r_{\text {sep }}=21 \mathrm{~mm}$, as well as $T_{i s e p}$. At $\Delta r_{\text {sep }}=21 \mathrm{~mm}, T_{i f i l} \approx 80-110 \mathrm{eV}$, which is about $50-70 \%$ of $T_{i s e p}$ and $3-4 T_{i}$. The finding $T_{i f i l} \approx 3-4 T_{i}$ is not extreme. Similar filament-to-background ion temperature ratios in the SOL have been observed in non-linear gyrofluid simulations of AUG L-mode plasmas (Ref. [40] and section 4). It is also worth mentioning that the electron temperature in filaments in the SOL of DIII-D, estimated from a multi-tip probe, was almost 3 times the background electron temperature [8]. Estimated $T_{i \text { fil }}$ tends to increase with $j_{\text {sat fil }}$, though the error bars are quite large relative to the observed variation of $T_{i \text { fil }}$. From $T_{i \text { fil }}$ and $j_{\text {sat fil }}$ one can deduce the relation between the amplitude of the fluctuations of the plasma density and the ion temperature. Assuming $n_{e f i l} \propto j_{\text {sat fil }} / \sqrt{T_{i f i l}}$, an unconstrained non-linear least-squares fit to the measured data gives $T_{i f i l} \propto n_{e f i l}^{0.53 \pm 0.13}$, i.e. the dynamic range of the ion temperature fluctuations is smaller than that of the electron density fluctuations.

The observations in Fig. 8 lead to the question with what radial speed $v_{r}$ a filament needs to travel across the SOL to arrive at $\Delta r_{\text {sep }}=21 \mathrm{~mm}$ with $T_{i \text { fil }}=50-70 \%$ of $T_{i s e p}$. In order to estimate $v_{r}$ we use a fluid model of the parallel filament transport described in [14]. In the model, the filament transport is described in the filament frame of reference by the temporal evolution of a Gaussian structure in which the initial particle and energy content decreases due to parallel losses to the divertor targets along open field lines. The model was previously employed to study the transport of ELM filaments in JET (e.g. [14, 26]) and AUG $([17,41])$. Once the initial filament temperatures and density are specified, their time evolution due to parallel transport to the nearest surface can be calculated. Concerning the question of the initial filament parameters, observations in AUG [21] and elsewhere (e.g. [42]) indicate that L-mode filaments form near the separatrix. Therefore, the values $T_{i \text { sep }}=150 \mathrm{eV}, T_{\text {esep }}=50 \mathrm{eV}$ and $n_{\text {esep }}=2.5 \times 10^{18} \mathrm{~m}^{-3}$ from Sec. 2 are assumed for the initial filament temperatures and electron density. Relatively high separatrix collisionality (Sec. 2) justifies the use of the fluid description of the parallel filament transport. In the model, 
temporal and radial evolution of the filament parameters are coupled through $v_{r}$. For simplicity, $v_{r}$ is assumed to be independent of $\Delta r_{\text {sep }}$. As illustrated in Fig. 9, $v_{r}$ is adjusted to bring modelled $T_{i \text { fil }}$ into agreement with the RFA measurements. As can be seen from Fig. 8, the model requires $v_{r} \approx 400-1000 \mathrm{~m} \mathrm{~s}^{-1}$ on average, which compares favourably with previous experimental estimates in L-mode plasmas $\left(\sim 500 \mathrm{~m} \mathrm{~s}^{-1}\right.$ in Alcator C-Mod [42], 330$2600 \mathrm{~m} \mathrm{~s}^{-1}$ in DIII-D [8, 43], 500-1500 m s$~^{-1}$ in MAST [44], $400 \mathrm{~m} \mathrm{~s}^{-1}$ in NSTX [45] and $\sim 500-2500 \mathrm{~m} \mathrm{~s}^{-1}$ in QUEST [46]). The increase of $v_{r}$ with the fluctuation amplitude (though marginal given the uncertainty in $v_{r}$ ) suggests that the mechanism underlying the radial motion of L-mode filaments in the SOL is the interchange drive [7]. For $v_{r} \approx 400-1000$ $\mathrm{m} \mathrm{s}^{-1}$, the estimated radial filament size $\Delta_{r} \approx v_{r} \Delta t \approx 1 \mathrm{~cm}$ (with $\Delta t \approx 15 \mu$ s from Fig. 4) is consistent with earlier observations ([1,2] and references therein).

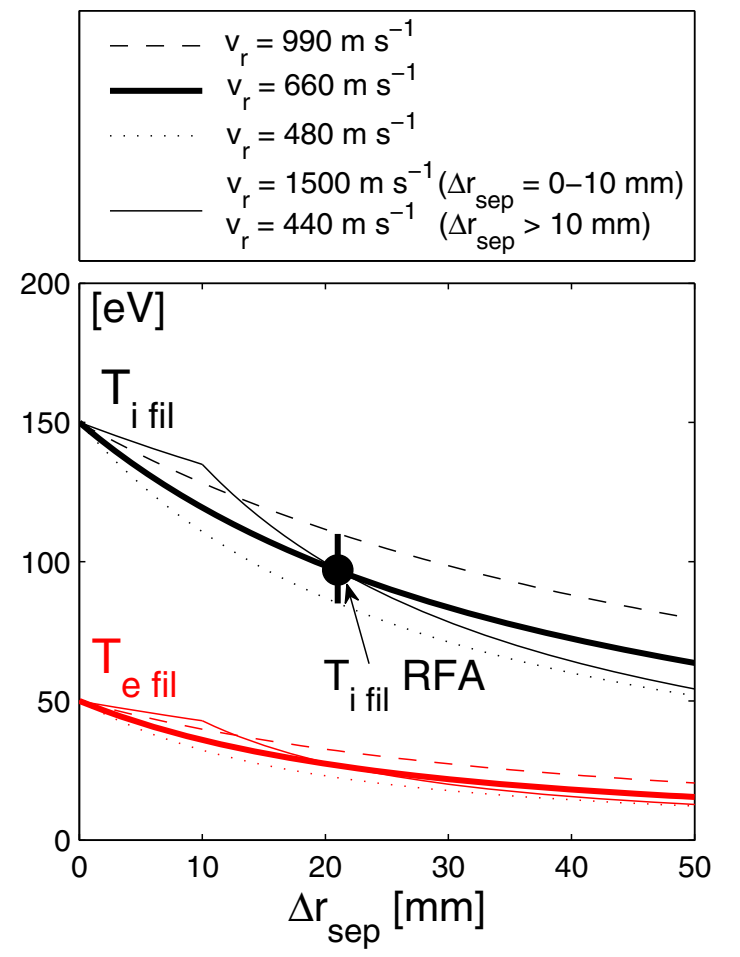

Figure 9. Radial evolution of the filament ion and electron temperatures obtained from the fluid parallel loss model. The filament is launched from the separatrix. Radially constant $v_{r}$ is adjusted to bring simulated and measured $T_{i \text { fil }}=97 \pm 12 \mathrm{eV}$ at $\Delta r_{\text {sep }}=21 \mathrm{~mm}$ into agreement. Also illustrated are the temperature profiles obtained for a non-constant form of $v_{r}$.

For $v_{r}=400-1000 \mathrm{~m} \mathrm{~s}^{-1}$, the simulations predict the filament stored energy normalized to its initial energy $W / W_{0} \approx 40-60 \%$ at $\Delta r_{\text {sep }}=21 \mathrm{~mm}$. For the same range of $v_{r}$, near the outer limiter radius $W / W_{0}$ falls in the range of $15-30 \%$ with the filament temperatures of up to $\sim 50 \%$ of the initial temperatures. 
A note is given concerning the assumption of radially constant $v_{r}$. Some experiments indicate that L-mode filaments decelerate as they propagate outwards and shrink in size [8, 43]. In Ref. [43], the observed $v_{r}=2600 \mathrm{~m} \mathrm{~s}^{-1}$ at $\Delta r_{\text {sep }}=0.5 \mathrm{~cm}, 1000 \mathrm{~m} \mathrm{~s}^{-1}$ at $\Delta r_{\text {sep }}=5 \mathrm{~cm}$ and $330 \mathrm{~m} \mathrm{~s}^{-1}$ at $\Delta r_{\text {sep }}=10 \mathrm{~cm}$. This indicates that for $\Delta r_{\text {sep }}=0-2 \mathrm{~cm}, v_{r}$ varies only by about $15 \%$, which is fairly consistent with our assumption of constant $v_{r}$ in that region. Nevertheless, to illustrate how the radial variation of $v_{r}$ affects the predicted filament temperatures in the SOL, the simulation from Fig. 9 has been reproduced assuming that the filaments decelerate in a stepwise manner with increasing the separatrix distance. For example, imposing arbitrarily chosen $v_{r}=1500 \mathrm{~m} \mathrm{~s}^{-1}$ for $\Delta r_{\text {sep }}=0-10 \mathrm{~mm}$, the model requires $v_{r}=440 \mathrm{~m} \mathrm{~s}^{-1}$ for $\Delta r_{\text {sep }}>10 \mathrm{~mm}$ to match $T_{i f i l}=97 \mathrm{eV}$ measured by RFA at $\Delta r_{\text {sep }}=21 \mathrm{~mm}$, as shown in Fig. 9. Finally a comment is made regarding the uncertainty in $T_{\text {esep }}$ mentioned in Sec. 2. If $T_{\text {esep }}=T_{\text {isep }}=150 \mathrm{eV}$ were assumed in all simulations, $v_{r}$ required to match the RFA measurements of $T_{i f i l}$ would be only by $\sim 10 \%$ higher. This is because of weak thermal coupling of filament ions and electrons. Therefore, the uncertainty in $T_{\text {esep }}$ leads to a slight but not decisive difference in the predicted range of $v_{r}$.

Before proceeding further, a remark is given to the standard model used to interpret the RFA measurements (e.g. [28, 47-49]). The model assumes that ions in the SOL are thermalized, so that the unperturbed 'SOL ion temperature' can be obtained from the $I-V$ characteristics measured by both sides of a bidirectional RFA using the aforementioned calibration [29]. The present study distinguishes between the ion temperature in the filaments and that of the background plasma, showing that the first can be considerably higher compared with the latter. This raises a question if it actually makes sense to apply the standard RFA model in the presence of a hot filament ions population. Presumably, at high $V_{g 1}$ the current to the collector becomes dominated by more energetic filament ions and the standard RFA model can yield a falsely high effective ion temperature. It is beyond the scope of this contribution to address these effects in detail. However, as illustrative example, the algorithm from Sec. 3.2 applied on the i-side RFA $I-V$ characteristics measured at $\Delta r_{\text {sep }}=21 \mathrm{~mm}$ yields the $e$-folding voltage of $52 \mathrm{eV}$. This is a factor 1.5-2 lower than $T_{i f i l}$, but yet a factor 2 higher compared with the estimated background ion temperature. Note that $T_{i^{*}}^{i-s i d e}=52 \mathrm{eV}$ would 
imply $T_{i^{*}}^{i-s i d e} / T_{i^{*}}^{e-s i d e} \approx 2.3$ which is significantly larger compared with $T_{i^{*}}^{i-s i d e} / T_{i^{*}}^{e-s i d e} \approx 1.3$, expected due to previously mentioned flow effects. It is worth noticing that $T_{i^{*}}^{i-s i d e} / T_{i^{*}}^{e-s i d e} \approx 1.6-2.2$ measured by a bi-directional RFA located at the top low-field side in JET was also larger compared with $T_{i^{*}}^{i-s i d e} / T_{i^{*}}^{e-s i d e} \approx 1.5$ predicted by the kinetic theory [29] for the measured values of $M_{/ /}$and $T_{i} / T_{e}$ [32]. Our remark is not intended to criticize earlier RFA measurements (insufficient sampling frequencies in these experiments smeared out most filaments and did not allow addressing the effect of a blobby transport on RFA $T_{i}$ measurements). It is intended to point out that the standard RFA model, though simple and convenient, can yield erroneous results in a turbulent plasma.

\section{Simulations of $T_{i}$ fil measurements}

In absence of instruments for fast SOL $T_{i}$ measurements, the simulations provide the only means of testing the reliability of the data analysis method used in the previous section to obtain $T_{i \text { fil }}$.

The time traces of fluctuating ion and electron temperatures $\left(T_{i, e}\right)$ and plasma density $n$ are generated by the global nonlinear three-dimensional electromagnetic gyrofluid turbulence code GEMR. The details of the code may be found in the references [50-55]. The code has been recently used to study the effect of the electron temperature fluctuations on the Langmuir probe measurements $[40,56]$. The code solves the first six moments of the gyrokinetic equation for ions and electrons in a circular flux surface geometry, using a consistent treatment of the energy conservation [51]. The simulation domain comprises $r / a=1 \pm 0.06$, i.e. both, the edge of the confined plasma and the SOL. Background midpedestal temperatures $T_{i}=180 \mathrm{eV}$ and $T_{e}=150 \mathrm{eV}$ and the plasma density $n=1.25 \times 10^{19} \mathrm{~m}^{-}$ ${ }^{3}$, with the perpendicular temperature and density gradient lengths, $L_{T \perp}=3 \mathrm{~cm}$ and $L_{n \perp}=6 \mathrm{~cm}$, respectively, are chosen arbitrarily as input parameters. Deuterons and a background magnetic field of $B=2 \mathrm{~T}$ are assumed. The plasma major radius $R=1.65 \mathrm{~m}$ and the aspect ratio $R / a=3.3$ are roughly consistent with typical AUG plasma parameters. Since the attempt is not to make a quantitative comparison with the experimental data, the input parameters can be considered a good enough approximation to an AUG-like L-mode plasma. 
The averaged grid size perpendicular to the magnetic field is $(1.06 \times 1.39) \rho_{s}$ with the gyroradius $\rho_{s}=0.88 \mathrm{~mm}$.

A synthetic RFA probe is located near the outboard midplane, $15.2 \mathrm{~mm}\left(\approx 17.2 \rho_{s}\right)$ outside the separatrix. The time traces of $T_{i}, T_{e}$ and $n$ are monitored over $9 \mathrm{~ms}$. A snapshot of $T_{i}, T_{e}$ and $n$ in the poloidal $(R-z)$ plane is depicted in Fig. 10 (animation online). For simplicity, $V_{g 1}$ applied to the probe is varied in this time interval from 0 to $270 \mathrm{~V}$, corresponding to the voltage range used in the present experiment. Since the filaments emerge randomly, the same results would be obtained if $V_{g 1}$ were swept at higher frequencies. The ion current density $j_{\text {sat }}$ is evaluated as enc and rescaled arbitrarily to roughly match the magnitude of $j_{\text {sat }}^{i-s i d e}$ measured in experiment.

$\mathrm{t}=2 \mathrm{~ms}$

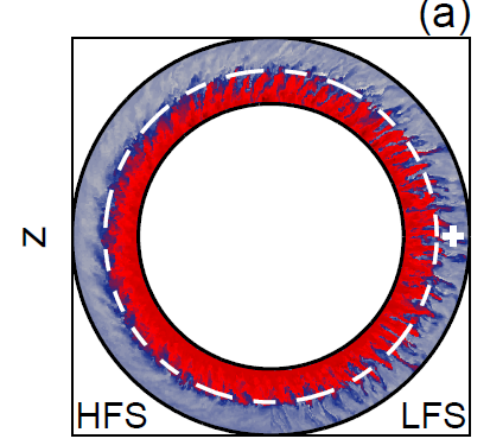

$\mathrm{R}$

(a)

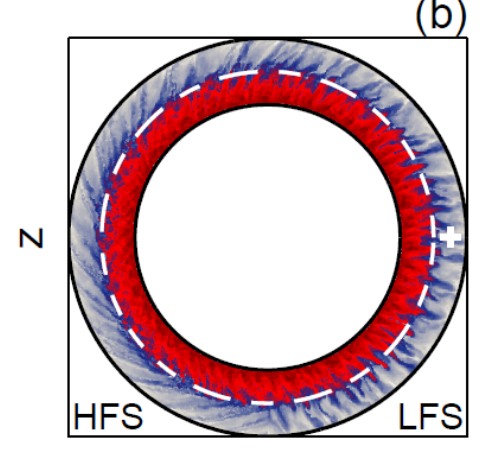

$\mathrm{R}$ (b)

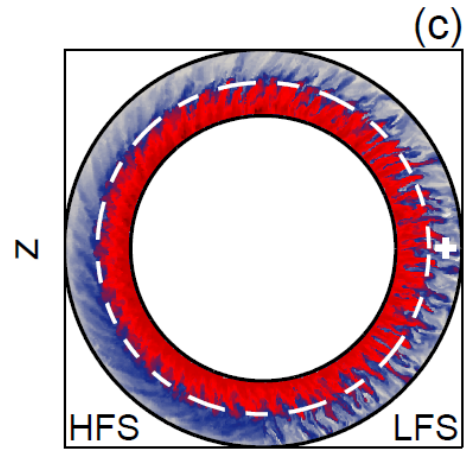

$\mathrm{R}$

Figure 10. Poloidal cross-section of (a) ion and (b) electron temperatures and (c) plasma density from the GEMR simulation (animation online) [please insert here URL to animation.gif]. For the sake of clarity, the radial dimension of the simulation domain $r / a=$ $1 \pm 0.06$ is expanded. The cross indicates an RFA sensor. Dashed: last closed magnetic surface. HFS/LFS: high field side / low field side.

As depicted in Fig. 2, the ions approaching the RFA slit plate gain the parallel energy of $e\left(V_{\text {sheath }}+V_{s p}\right)$ in the Debye sheath, from which $e V_{s p}$ is removed as they proceed to grid 1. If a full $\mathrm{I}-\mathrm{V}$ characteristic was acquired for individual filaments, the decaying part of the characteristic would be shifted to higher $V_{g 1}$ by $V_{\text {sheath }}$, and $I_{c \text { fil }}$ would be constant for $V_{g 1}<V_{\text {sheath }}$. In the present experiment, however, each $I-V$ characteristic comprises a number 
of filaments, which might be associated to different values of $V_{\text {sheath }}$. The variation of $V_{\text {sheath }}$ in individual filaments can contribute to the scatter of the data points in the $I-V$ characteristic and can make the error of $T_{i \text { fil }}$ measurements larger. Therefore, in addition to the fluctuations of temperatures and density, the fluctuations of $V_{\text {sheath }}$ have to be considered if the test is to be realistic. The expression

$$
V_{\text {sheath }}=-0.5 T_{e} \ln \left[2 \pi \frac{m_{e}}{m_{i}}\left(1+\frac{T_{i}}{T_{e}}\right)\left(1-\delta_{\text {see }}\right)^{-2}\right] \text {, }
$$

available in many sources (e.g. [38]), is used to obtain $V_{\text {sheath }}$ from the simulated time series of $T_{i}$ and $T_{e}$. The lack of measurements of the secondary electron emission coefficient $\delta_{\text {see }}$ from the RFA slit plate introduces considerable freedom in $V_{\text {sheath }}$. However, as can be seen in Fig. 8, $I_{c \text { fil }}$ tends to decrease with increasing $V_{g 1}$ for the range of voltages at which the filament are captured. The same tendency is seen in other filament ion I-V characteristics. This observation suggests that $V_{\text {sheath }}$ is of the order of tens of volts when the filaments strike the probe (data scatter and the absence of $I_{c \text { fil }}$ measurements at lowest $V_{g 1}$ does not allow quantifying $V_{\text {sheath }}$ more precisely). In order to obtain the fluctuations of $V_{\text {sheath }}$ of this order from the simulated ion and electron temperatures, we assume arbitrarily $\delta_{\text {see }}=0.8$ in Eq.(1). It is at least partially convincing that similar $\delta_{\text {see }}$ was observed in very recent experiments in the Tore Supra tokamak under various conditions [57]. The collector current is evaluated as

$$
\begin{aligned}
& I_{c}=j_{\text {sat }} \text { for } V_{g 1}<V_{\text {sheath }}, \\
& I_{c}=j_{\text {sat }} \exp \left(-\frac{V_{g 1}-V_{\text {sheath }}}{T_{i}}\right) \text { for } V_{g 1}>V_{\text {sheath }},
\end{aligned}
$$

assuming Maxwellian ions [28] and with $V_{\text {sheath }}$ given by Eq.(1). For the sake of simplicity we have neglected the attenuation of the incident ion current by the RFA grids and by the slit plate aperture. Numerical studies suggest that the attenuation has only weak influence on $T_{i}$ measurements [16]. For illustrative purpose, $I_{c}$ is also evaluated neglecting $V_{\text {sheath }}$. 
The time traces of $T_{i}, T_{e}, n, j_{s a t}, V_{g 1}, V_{\text {sheath }}$ and $I_{c}$ are plotted in Fig. 11. The conditionally sampled bursts of $j_{\text {sat }}$ from GEMR plotted in Fig. 12 are asymmetric around the maximum current (albeit with a factor 2 shorter duration) which is consistent with experimental observation from Fig. 4. Moreover, the relative fluctuation level (1.09), skewness (1.86) and excess kurtosis (4.19) of the simulated $j_{\text {sat }}$ signal are reasonably close to those of $j_{\text {sat }}^{i-s i d e}$. These similarities provide some confidence that the fluctuations in the SOL are realistically simulated.
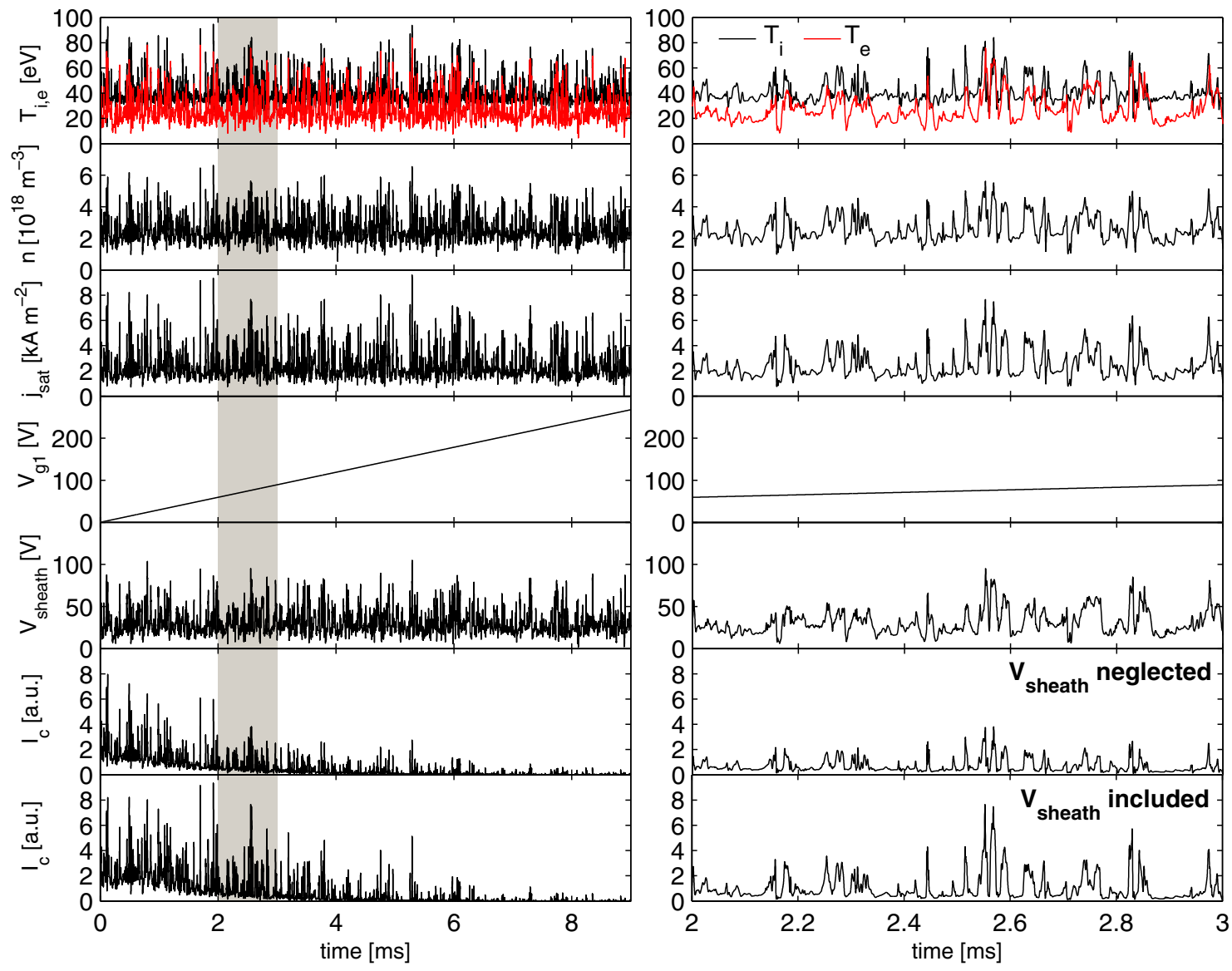

Figure 11. Left: Time traces of the SOL ion and electron temperatures and the plasma density obtained from the gyrofluid turbulence simulation and the voltage imposed to the ion repelling grid $V_{g I}$. The time traces of $j_{\text {sat }}, V_{\text {sheath }}$ and $I_{c}$ measured by a synthetic $R F A$ are evaluated as described in the text. Right: Expanded time traces for $t=2-3 \mathrm{~ms}$ (shaded). 


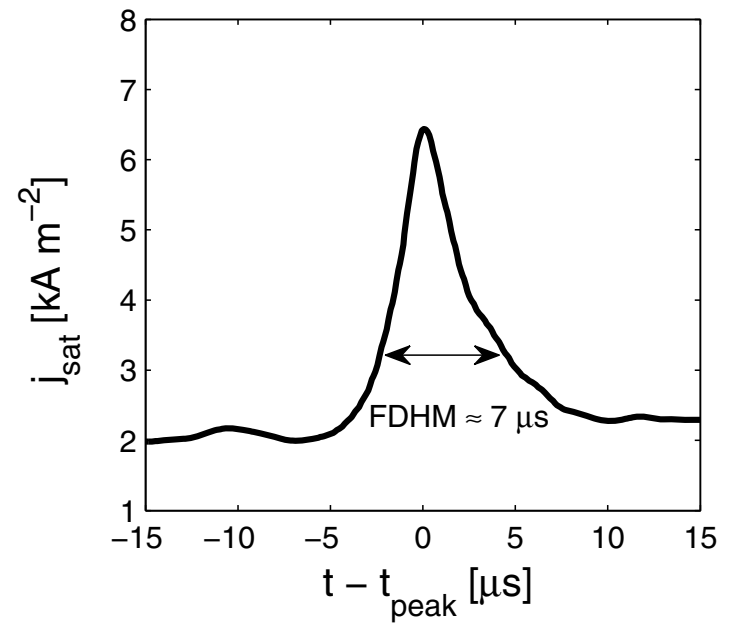

Figure 12. Time evolution of the largeamplitude bursts $(>3 \sigma)$ of $j_{\text {sat }}$ obtained by averaging 32 events captured $15.2 \mathrm{~mm}$ outside the separatrix in GEMR simulations. Also indicated is the full duration at half maximum.

The filament ion temperature is obtained from the time traces of $j_{s a t}, V_{g 1}$ and $I_{c}$ in the same way as in experiment. Included in the analysis are the filaments above the detection threshold $\bar{j}_{\text {sat }}+\sigma\left(j_{\text {sat }}\right)=3.4 \mathrm{kA} \mathrm{m}^{-2}$. Individual ion $I-V$ characteristics, containing a similar number of filaments as in experiment, obtained for the case of $V_{\text {sheath }}\left(\delta_{\text {see }}=0.8\right)$ and $V_{\text {sheath }}=0$ are shown in Fig. 13. In either case, the slope of the characteristics flattens (i.e. $T_{i f i l}$ increases) as $j_{\text {sat fil }}$ increases, which is consistent with the observation from Fig. 8. Also plotted in Fig. 13 are exponential fits to individual $I-V$ characteristics. In the case when the fluctuations of $V_{\text {sheath }}$ taken in account, the range of $V_{g 1}$ included in the fit is restricted to $V_{g 1}>70 \mathrm{~V}$ as in experiment. In Fig. 14, $T_{i f i l}$ obtained from the fit is plotted against the mean value of $T_{i f i l}$ of the filaments in each $I-V$ characteristic. Vertical error bars coincide with the confidence interval of the exponential fit, whilst the horizontal error bars coincide with the standard deviation of $T_{i \text { fil }}$ values of the filaments in each $I-V$ characteristic. In the idealized case without the fluctuations of $V_{\text {sheath }}$, the match between the simulated and the conditionally sampled $T_{i f i l}$ is excellent. The cause is a strong correlation of temperatures and density fluctuations (as can be seen in Fig. 11 and typically observed in GEMR simulations) due to the $\mathbf{E} \times \mathbf{B}$ advection. When the fluctuations of $V_{\text {sheath }}$ are taken into account, the match becomes somewhat worse, though $T_{i \text { fil }}$ is still reconstructed with a good precision. Trials with a different width of the $j_{\text {sat fil }}$ averaging window revealed that the reconstruction method is insensitive to this parameter. 

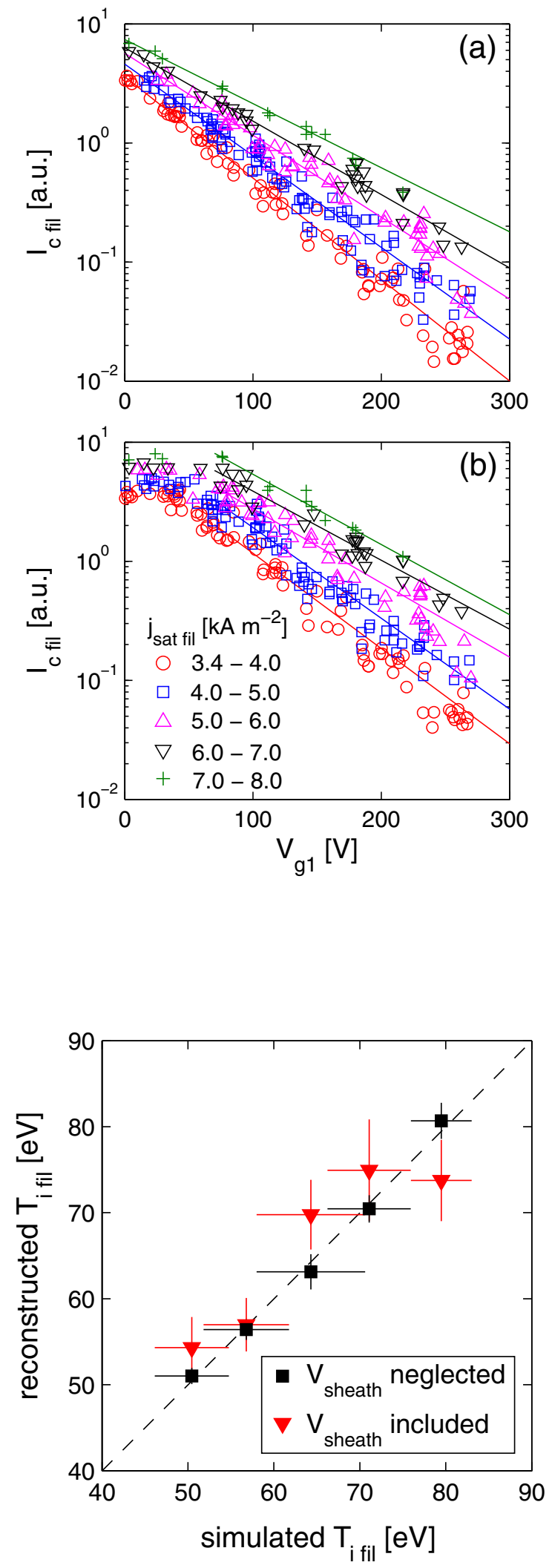

Figure 13. Semi-logarithmic plot of the collector current against the ion repelling voltage measured by a synthetic RFA. Data points corresponding to similar $j_{\text {sat fil }}$ are colour coded. Full lines: exponential fit to individual filament ion I-V characteristics. The collector current is obtained from the simulation data (a) neglecting and (b) including the fluctuations of $V_{\text {sheath }}$.

Figure 14. The filament ion temperature reconstructed from the GEMR simulation using conditional averaging plotted against the mean of the $T_{i f i l}$ values corresponding to the data points included in individual I-V characteristics. Results obtained neglecting and including the fluctuations of $V_{\text {sheath }}$ are shown. 


\section{Summary}

Ion energies in the L-mode turbulence filaments have been measured in the ASDEX Upgrade SOL using a bi-directional retarding field analyzer located above the outer midplane. A difference in the dynamic behavior of the ion current fluctuations is seen from the time traces measured by each of the two analyzers. Large-amplitude fluctuations of the ion current are observed by the analyzer viewing the outer midplane. These fluctuations are associated with plasma filaments ejected into the SOL on the plasma outboard side and passing the probe. The ion current fluctuations measured by the analyzer connected magnetically to the inner divertor are almost normally distributed. At $40 \rightarrow 20 \mathrm{~mm}$ outside the separatrix the RFA measures the background ion temperature $T_{i} \approx 20 \rightarrow 30 \mathrm{eV}$, corresponding to $2-3 T_{e}$. At $\Delta r_{\text {sep }}=21 \mathrm{~mm}$, the ion temperature in the turbulence filaments $T_{i \text { fil }}$ is about $80-110 \mathrm{eV}$ which is $3-4 T_{i}$ and $50-70 \%$ of the ion temperature at the separatrix obtained from the spectroscopic measurements. The measured $T_{i f i l}$ is reproduced by a fluid model of the parallel filament transport assuming $v_{r}=400-1000 \mathrm{~m} \mathrm{~s}^{-1}$ in the near SOL, i.e. without resorting to extreme assumptions about the radial filament propagation speed. Filaments propagating across the SOL at that radial speed carry $15-30 \%$ of their initial energy to the outboard limiters. The analysis of synthetic RFA measurements obtained from gyrofluid turbulence simulations shows that the conditional sampling of RFA $I-V$ characteristics allows for reasonably accurate measurements of $T_{i \text { fil }}$.

\section{Acknowledgements}

We wish to thank G. D. Conway, J. P. Gunn, B. Nold, R. A. Pitts and U. Stroth and the two anonymous referees for helpful comments. We are grateful to B. Scott (GEMR code) and D. Moulton (script of the parallel loss model). F.P. Gennrich and A. Kendl were supported by the Austrian Science Fund (FWF) Y398.

\section{References}

[1] S. J. Zweben et al., Plasma Phys. Control. Fusion 49 (2007) S1.

[2] D. A. D’Ippolito, J. R. Myra and S. J. Zweben, Phys. Plasmas 18 (2011) 060501. 
[3] B. LaBombard et al., Nucl. Fusion 44 (2004) 1047.

[4] N. Asakura, J. Nucl. Mater. 363-365 (2007) 41.

[5] R. A. Pitts, J. Horacek, W. Fundamenski, O. E. Garcia, A. H. Nielsen, M. Wischmeier, V. Naulin, J. Juul Rasmussen, J. Nucl. Mater. 363-365 (2007) 505.

[6] J. P. Gunn et al., J. Nucl. Mater. 363-365 (2007) 484.

[7] O. E. Garcia et al., Phys. Plasmas 13 (2006) 082309.

[8] J. A. Boedo et al., Phys. Plasmas 10 (2003) 1670.

[9] R. A. Pitts et al., J. Nucl. Mater. S415 (2011) S957.

[10] S. Carpentier et al., J. Nucl. Mater. S415 (2011) 165.

[11] J. Stockel et al., Problems of Atomic Science and Technology, 2006, No. 6. Series: Plasma Physics (12), p. 19-23, http://vant.kipt.kharkov.ua/CONTENTS/CONTENTS_2006_6.html

[12] I. S. Nedzelskiy, C. Silva, P. Duarte, and H. Fernandes, Rev. Sci. Instrum. 82 (2011) 043505 .

[13] A. Kallenbach et al., Nucl. Fusion 51 (2011) 094012.

[14] W. Fundamenski et al., Plasma Phys. Control. Fusion 48 (2006) 109.

[15] J. Neuhauser et al., Plasma Phys. Control. Fusion 44 (2002) 855.

[16] M. Kočan et al., Rev. Sci. Instrum. 79 (2008) 073502.

[17] M. Kočan et al., Plasma Phys. Control. Fusion 53 (2011) 065002.

[18] N. Ben Ayed et al., Plasma Phys. Control. Fusion 51 (2009) 035016.

[19] O. E. Garcia et al., Plasma Phys. Control. Fusion 48 (2006) L1-L10.

[20] O. E. Garcia, J. Horacek, R. A. Pitts, A. H. Nielsen, W. Fundamenski, V. Naulin and J. Juul Rasmussen, Nucl. Fusion 47 (2007) 667.

[21] B. Nold et al., Plasma Phys. Control. Fusion 52 (2010) 065005.

[22] J. Boedo, J. Nucl. Mater. 390-391 (2009) 29.

[23] M. Kočan and J. P. Gunn, Plasma Phys. Control. Fusion 52 (2010) 045010.

[24] N. Fedorczak et al., J. Nucl. Mater. S415 (2011) S467.

[25] G. Birkenmeier, M. Ramisch, P. Manz, B. Nold, and U. Stroth, Phys. Rev. Lett. 107 (2011) 025001. 
[26] R. A. Pitts et al., Nucl. Fusion 46 (2006) 82.

[27] R. A. Pitts, P. Andrew, G. Arnoux, T. Eich, W. Fundamenski, A. Huber, C. Silva, D. Tskhakaya and JET EFDA Contributors, Nucl. Fusion 47 (2007) 1437.

[28] A. S. Wan, T. F. Yang, B. Lipschultz and B. LaBombard, Rev. Sci. Instrum. 57 (1986) 1542.

[29] F. Valsaque et al., Phys. Plasmas 9 (2002) 1806.

[30] M. Kočan and J. P. Gunn, Plasma Phys. Control. Fusion 53 (2011) 085016.

[31] I.H. Hutchinson, Principles of Plasma Diagnostics, second edition, Cambridge University Press (2002).

[32] R. A. Pitts, I. Duran, S. K. Erents, J. Horacek, G. F. Matthews and the JET EFDA Contributors, Proc. 30th EPS Conference on Contr. Fusion and Plasma Phys., St.

Petersburg, 7-11 July 2003 ECA Vol. 27A, P-2.84

[33] M. Kočan and J. P. Gunn, Proc. 36th EPS Conference on Plasma Phys. Sofia, June 29 - July 3, 2009 ECA Vol.33E, P-4.203 (2009).

[34] I.H Hutchinson, Phys Fluids B 3 (1991) 847.

[35] I.H. Hutchinson, Phys. Fluids 30 (1987) 3777.

[36] R. Dejarnac, J.P. Gunn, J. Stockel, J. Adamek, J. Brotankova and C. Ionita, Plasma Phys. Control. Fusion 49 (2007) 1791.

[37] M. Kočan et al., J. Nucl. Mater. S415 (2011) S1133.

[38] P.C. Stangeby, The Plasma Boundary in Magnetic Fusion Devices, Institute of Physical Publishing, Bristol, 2000.

[39] I. Furno et al., Phys. Plasmas 15 (2008) 055903.

[40] F. P. Gennrich and A. Kendl, Plasma Phys. Control. Fusion 54 (2011) 015012.

[41] M. Kočan et al., Nucl. Fusion 52 (2012) 023016.

[42] O. Grulke et al., Phys. Plasmas 13 (2006) 012306.

[43] J. A. Boedo et al., Phys. Plasmas 8 (2001) 4826.

[44] B. D. Dudson et al., Plasma Phys. Control. Fusion 50 (2008) 124012.

[45] J. R. Myra et al., Phys. Plasmas 13 (2006) 092509.

[46] R. Ogata et al., Phys. Plasmas 18 (2011) 092306. 
[47] R. A. Pitts, Ph.D. thesis, University of London, 1991.

[48] H. Y. Guo, G. F. Matthews, S. J. Davies, S. K. Erents, L. D. Horton, R. D. Monk, and P. C. Stangeby, Contrib. Plasma Phys. 36S (1996) 81.

[49] M. Kočan et al., Plasma Phys. Control. Fusion 50 (2008) 125009.

[50] B. D. Scott, Phys. Plasmas 8 (2001) 447.

[51] B. D. Scott, Phys. Plasmas 12 (2005) 102307.

[52] T. T. Ribeiro and B. D. Scott, Plasma Phys. Control. Fusion 47 (2005) 1657.

[53] B. D. Scott, Contrib. Plasma Phys. 46 (2006) 714.

[54] T. T. Ribeiro and B. D. Scott, Plasma Phys. Control. Fusion 50 (2008) 055007.

[55] A. Kendl, B. D. Scott and T. Ribeiro, Phys. Plasmas 17 (2010) 072302.

[56] B. Nold et al., Influence of temperature fluctuations on plasma turbulence investigations with Langmuir probes, submitted to New Journal of Physics 2011.

[57] J. P. Gunn et al., Scrape-off Layer Power Flux Measurements in the Tore Supra Tokamak, in preparation for the 20th International Conference on Plasma Surface Interactions, Aachen, Germany, 21- 25 May 2012. 